\title{
The thin white line: Adaptation suggests a common neural mechanism for judgements of Asian and Caucasian body size
}

\author{
Lewis Gould-Fensom ${ }^{1,2}$, Chrystalle BY Tan ${ }^{3}$, Kevin R Brooks ${ }^{1,4}$, Jonathan Mond ${ }^{5,6}$, Richard J \\ Stevenson $^{1,4}$, Ian D Stephen ${ }^{1,4 *}$
}

1 Department of Psychology, Macquarie University, Sydney, New South Wales, Australia

2 Department of Psychology, University of Melbourne, Melbourne, Victoria, Australia

3 Faculty of Medicine and Health Sciences, Universiti Malaysia Sabah, Kota Kinabalu, Malaysia

4 Perception in Action Research Centre (PARC), Faculty of Human Sciences, Macquarie University, Sydney, New South Wales, Australia

5 Centre for Rural Health, University of Tasmania, Launceston, Tasmania, Australia

6 Centre for Health Research, School of Medicine, Western Sydney University, Sydney, New South Wales, Australia

* Correspondence:

Ian D Stephen

ian.stephen@mq.edu.au

Keywords: Body perception, Visual adaptation, Visual aftereffects, Cross-cultural, Body image, Body size.
Abstract
Visual adaptation has been proposed as a mechanism linking viewing images of thin women's bodies with body size and shape misperception (BSSM). Non-Caucasian populations appear less susceptible to BSSM, possibly because adaptation to thin Caucasian bodies in Western media may not fully transfer to own-race bodies. Experiment 1 used a cross-adaptation paradigm to examine the transfer of body size aftereffects across races. Large aftereffects were found in the predicted directions for all conditions. The strength of aftereffects was statistically equivalent when the race of test stimuli was congruent vs incongruent with the race of adaptation stimuli, suggesting complete transfer of aftereffects across races. Experiment 2 used a contingent-adaptation paradigm, finding that simultaneous adaptation to wide Asian and narrow Caucasian women's bodies (or vice versa) results in no significant aftereffects for either congruent or incongruent conditions, and statistically 
equivalent results for each. Equal and opposite adaptation effects may therefore transfer completely across races, cancelling each other out. This suggests that body size is encoded by a race-general neural mechanism. Unexpectedly, Asian observers showed reduced body size aftereffects compared to Caucasian observers, regardless of the race of stimulus bodies, perhaps helping to explain why Asian populations appear less susceptible to BSSM.

\section{Introduction}

Body size and shape misperception (BSSM) - a phenomenon in which some individuals overestimate or underestimate their body size - affects a large and growing segment of the population of Western countries (Powell et al., 2010; Quick et al., 2015), and is becoming increasingly common in Asian societies and around the world (Wardle et al., 2006). Body size overestimation is associated with high levels of body dissatisfaction, anxiety and depression, and is a risk factor for the development of eating disorders and compulsive exercise behaviour, particularly in young women (Bould et al., 2018; Griffiths et al., 2016). In Malaysia, a rapidly-developing Southeast Asian country, 20.5\% of underweight young women in a large epidemiological study overestimated their body size (Khor et al., 2009), while a large longitudinal study of young people in 24 Western countries found that over $40 \%$ of non-overweight young women overestimated their body size (Quick et al., 2014).

Meanwhile, approximately a quarter of overweight (BMI > 25) women and half of overweight men misperceive themselves as having the "right weight" (Yaemsiri et al., 2011), suggesting they may be underestimating their body size. This form of BSSM may be associated with reduced motivation to lose weight (Powell et al., 2010).

Explanations for body size overestimation have typically focussed on the role of the Western media's portrayal of idealised thin female bodies (Groesz et al., 2002). This connection between exposure to Western media and body image distortion is well established, with recent studies showing that the introduction of television into a region is associated with thinner ideal body size 
(Boothroyd et al., 2016), even when controlling for potential confounds such as nutritional status (Jucker et al., 2017). However, some evidence suggests that exposure to idealised bodies may have less pronounced effects on body dissatisfaction (which is affected by BSSM; Bould et al., 2018) for individuals of races not represented in the images (DeBraganza and Hausenblas, 2010).

Explanations for how exposure to idealised media portrayals of thin bodies leads to body image distortion have tended to focus on sociocultural mechanisms such as social comparison. However, there is evidence that mere exposure to a visual diet containing these images is sufficient to induce changes in body size preferences (Stephen and Perera, 2014) independent of cues to the social status of the individuals depicted (Boothroyd et al., 2012). Similarly, it has been suggested that body size underestimation by obese individuals may be attributable to a visual diet in which overweight and obese bodies are common, as overweight and obesity become more common in the population (Robinson and Kirkham, 2014).

Recently, it has been suggested that visual adaptation may offer a mechanism for the development of both over- and underestimation of body size (Challinor et al., 2017; Mohr et al., 2016; Sturman et al., 2017). Visual adaptation is a phenomenon whereby prolonged exposure to a particular stimulus (e.g. downward motion) causes adaptation of the associated neurons such that subsequently presented stimuli (stationary objects) appear to have properties opposite to those shown by the adapted stimulus (to be moving upwards). These visual adaptation effects are observed in a range of low-level properties of stimuli, including orientation, colour, depth, and spatial frequency (Thompson and Burr, 2009). More recently, visual adaptation has been demonstrated in higher-level stimulus properties, including the identity (Rhodes et al., 2011) and gender (Yang et al., 2011) of faces, and the size and shape of bodies (Mohr et al., 2016; Sturman et al., 2017). For bodies, exposure to wide (narrow) figures causes a change in perceived size such that subsequently viewed 
body images appear narrower (wider) than they are (Challinor et al., 2017). Hence, the point of subjective normality (PSN) moves towards the adaptation stimulus.

For faces, the extent of visual adaptation depends in part on the race of the stimuli. Participants exposed to distorted images of faces of one race (e.g. expanded Asian faces) show an aftereffect of perceived contraction that is larger when assessed using test faces of the same race than for test faces of a different race (e.g. Caucasian faces) - a phenomenon known as partial crossadaptation (Jaquet, Rhodes, \& Hayward, 2008). Further, when exposed to faces of different races distorted in opposite directions (e.g. expanded Asian and contracted Caucasian faces), participants show "contingent adaptation" - simultaneous visual adaptation in both races in opposite directions (e.g. subsequently presented Asian faces appeared contracted, while Caucasian faces appeared expanded) (Gwinn and Brooks, 2015; Jaquet et al., 2008). This suggests that faces of different races are encoded by partially-dissociated sets of neurons. Similar dissociations have also been demonstrated for the encoding of faces of different genders and ages (Yang et al., 2011).

Partial transfer of body size aftereffects and category-contingent body size adaptation have also been demonstrated. Brooks et al (2016) exposed participants to adaptation stimuli with a particular identity (e.g. wide other-identity bodies), showing aftereffects of reduced magnitude when tested using stimuli with identities different to the adaptation stimuli (e.g. their own body). Furthermore, participants exposed to different-identity bodies that have been distorted in opposite directions (e.g. wide own-identity and narrow other-identity bodies) show simultaneous aftereffects in opposite directions in each body identity (e.g. own-identity bodies appear narrower and otheridentity bodies appear wider than they are). This suggests that own- and other-identity bodies are encoded by partially dissociated neural populations (Brooks et al., 2016). It is not known, however, whether bodies of different races are encoded using independent or partially dissociated neural populations, or a single population that is not race-sensitive. If there were no transfer of body size 
aftereffects, or if transfer was only partial, it may provide an explanation for the reduced effects of viewing other- (compared to own-)race bodies on observers' body dissatisfaction (DeBraganza and Hausenblas, 2010), and may shed light on the observed lower rates of body image disturbance in cultures where western media promotes the thin ideal using Caucasian models to populations of other races. For example, exposure to mainstream television (which typically features primarily white bodies) had no effect on African American women's body image, where 


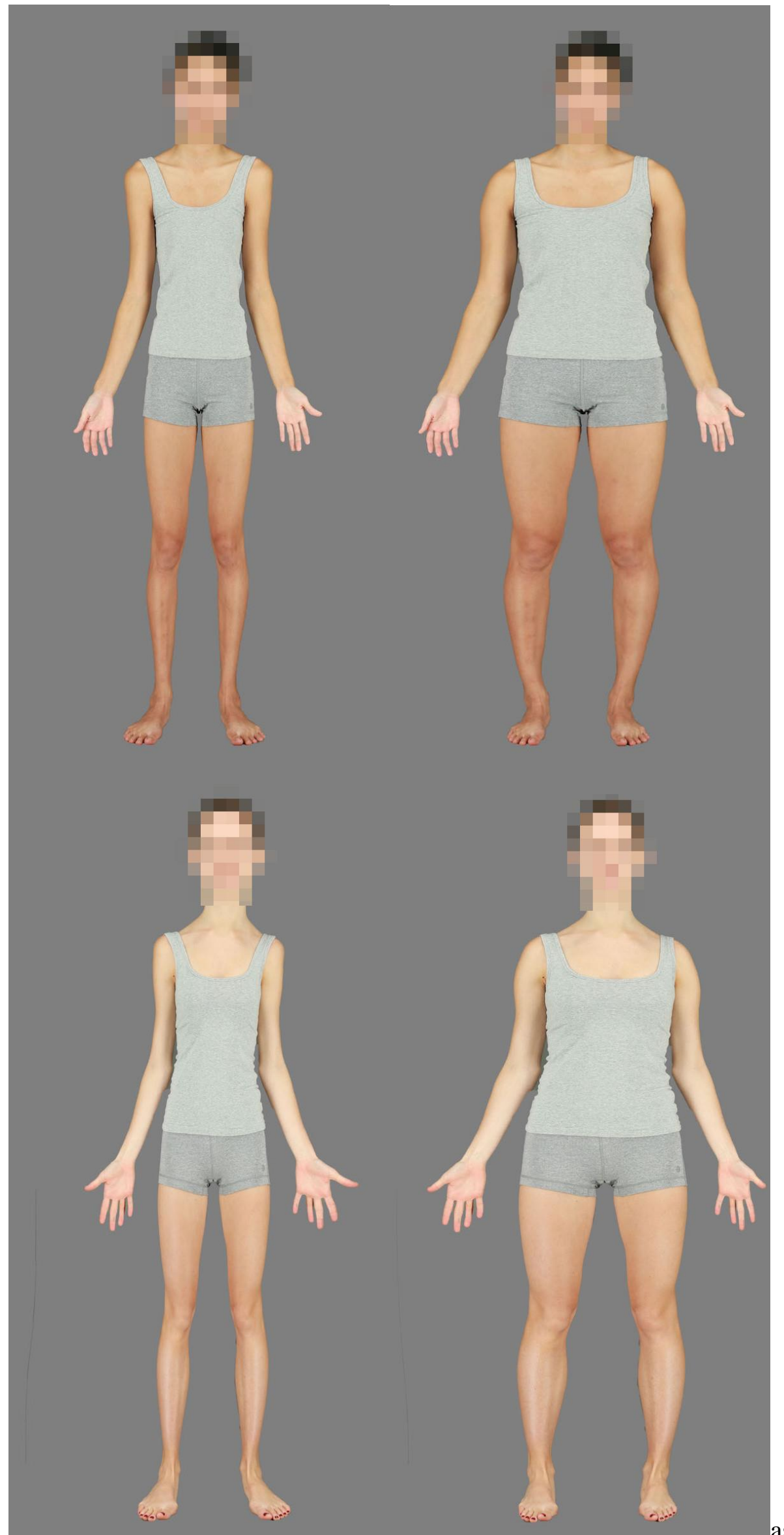

as exposure to Black-oriented

This is a provisional file, not the final typeset article 
media (which typically includes black bodies and larger body sizes) improved their body image (Schooler et al., 2004).

Here, we present two experiments that aim to determine the extent of transfer of body size aftereffects across bodies of different races, and thus to shed light on the nature of the neural populations encoding body size in bodies of different races. Based on the known dissociation of neural populations for the processing of facial distortion, we hypothesised that participants would show partial transfer of body size adaptation effects between bodies of different races.

\section{Methods}

All work was approved by both the Macquarie University Human Research Ethics Committee (MUHREC), as well as the Medical Ethics Committee of the Universiti Malaysia Sabah (MECUMS). All participants gave prior, informed consent in writing.

\subsection{Experiment 1}

Experiment 1 used a cross-adaptation paradigm to elucidate whether the neural populations encoding body size for Asian and Caucasian identities are common or independent. Participants were exposed either to Asian or Caucasian adaptation stimuli and tested with identities from both races. If independent race-specific neural populations are used for the encoding of body size for each race, we predict that aftereffects should be demonstrated when test bodies are from the same racial group as the adaptation stimuli (congruent conditions), but absent when the two are incongruent (i.e. zero transfer of the aftereffect). Alternatively, encoding of body size by a common, race-general population would predict equivalent aftereffect magnitudes for Asian and Caucasian test identities regardless of the race of adaptation stimuli (i.e. $100 \%$ transfer). An intermediate arrangement is also possible, such that that neural populations show partial overlap. This allows us to predict aftereffects 
for all test bodies, but that these should be stronger for Asian compared to Caucasian test identities following adaptation to Asian adaptation identities, and vice versa (partial transfer).

\subsubsection{Method}

\subsubsection{Participants}

One hundred and twenty seven female participants were recruited in Australia $(n=67)$ and Malaysia ( $n=60$ ), giving $95 \%$ power to detect a small-medium effect size for the hypothesised interaction in the planned ANOVA. Australia-based participants $\left(M_{a g e}=21.8, S D=5.23\right)$ self-identified as Caucasian, were primarily first-year undergraduates and received course credit for their participation. Malaysia-based participants $\left(M_{\text {age }}=24.6, S D=5.72\right)$, who self-identified as East/Southeast Asian, were a mix of family and friends of the researcher and students at the Universiti Malaysia Sabah, and were entered into a draw to win one of three RM50 gift cards for their participation. Two Australian and four Malaysian participants were excluded from the final analysis because they did not identify as 'Caucasian' (in Australia) or 'East/South East Asian' (in Malaysia). Additionally, five Australian participants were excluded from the final analysis because of technical problems during their participation sessions, leaving a total of 116 participants.

\subsubsection{Stimuli}

Colour, full-body photographs of Caucasian and Asian individuals were obtained from a database built for a larger study. Photographs were taken with participants wearing standard grey, tight-fitting shorts and singlet, standing in a Munsell N5 grey-painted photo booth, under standardised lighting and with all camera settings held constant (Sturman et al., 2017).

Twenty-two Asian and 22 Caucasian identities were used in this study. Height and weight measurements were taken and BMI (Asian $M=22.19, S D=3.14$; Caucasian $M=22.18, S D=3.22$ ) calculated, which were then used to match each Asian identity to a corresponding Caucasian identity. Images were resized to 450x900 pixels, and all identifying markings or tattoos were digitally 
removed using the clone stamp tool in Adobe Photoshop CS6. For each identity, the body (from the neck downward) was expanded horizontally by $50 \%$ and contracted by $50 \%$, using the Spherize tool in Photoshop. This created 2 images of the same identity that appeared to differ drastically in body fat. These were used as the adaptation stimuli (Figure 1).

A further 12 images, horizontally expanded or contracted in 5\% increments, up to and including $\pm 30 \%$, were created as the test stimuli to add to the original " $0 \%$ " image (Figure 2 ). The faces of each identity remained visible so that race could easily be discerned, but faces were left unaltered by the Spherize manipulation to avoid the possibility of confounding effects of face adaptation.

\subsubsection{Procedure}

Participants were pseudorandomly assigned to one of four adaptation condition to determine the adaptation stimuli with which they would be presented - Expanded Asian (A+), Expanded Caucasian (C+), Contracted Asian (A-), or Contracted Caucasian (C-). Participants provided their age, race and sex via a demographics questionnaire in Qualtrics, before proceeding to the practice phase. The practice phase consisted of a series of two Asian and two Caucasian test identities (each Asian test identity matched for initial BMI with a Caucasian test identity), presented one at a time in random order. A Method of Adjustment task programmed in Matlab R2017b with Psychtoolbox 3 was used. For each test identity, moving the mouse horizontally cycled through the 13 frames of the test identity's transformation. Participants were instructed that moving the mouse horizontally would change the appearance of the body (but were not told it was a width manipulation) and were asked to "make the body look as normal as possible", then click to save the data and move onto the next identity. The initial frame presented was randomised. Each trial was preceded by a beep to indicate that a response was required. 
Next, in the baseline test phase, participants were presented with a series of 20 test identities (10 Asian, 10 Caucasian, each Asian identity matched for initial BMI with a Caucasian identity), selected at random from the pool, one at a time in random order, and asked to make them appear as normal as possible.

In the adaptation test phase, participants were presented with a two-minute slide show of 10 identities according to their Condition (expanded or contracted Asian or Caucasian bodies). Each identity was presented six times, for a total duration of 12 seconds. Participants were not required to make a response and no beep sound was played. Following this initial adaptation, participants were presented with the same 20 test identities from the baseline phase (10 Asian, 10 Caucasian, matched on initial BMI), one at a time, in random order, and asked to make the bodies as normal as possible. Between each trial, a series of six "top up" adaptation stimuli were selected at random from the adaptation identities and presented for 1 second each (a total of 6 seconds) to ensure the maintenance of the adaptation effect (Brooks et al., 2016). Participants were asked to simply observe the top up stimuli without making a response.

\subsubsection{Data processing and analysis}

Baseline point of subjective normality (PSN) was calculated for each participant as the average amount of expansion/contraction chosen to make the bodies look as normal as possible across the baseline trials. Adapted PSN was calculated for each participant as the average amount of expansion/contraction chosen across the adaptation test phase trials. Change in PSN ( $\triangle \mathrm{PSN}$ ) was calculated by subtracting baseline PSN from adapted PSN, such that a positive (negative) value indicated that the participant's perception of normal body size had become wider (narrower) as a result of adaptation. This was used as the main dependent variable.

Separate one-sample t-tests were used to test for the presence of aftereffects in each of the four conditions for each of the test races. A 2 (race of test stimuli; within subjects) x 2 (race of 
adaptation stimuli; between subjects) x 2 (race of participant; between subjects) x 2 (direction of adaptation; expanded or contracted) mixed ANOVA was used to determine whether there was significant transfer of adaptation across bodies of different races, for participants of different races.

\subsubsection{Results}

The average $\triangle \mathrm{PSN}$ for each condition was found to be significantly different from 0 in the predicted direction (Figure 3; those exposed to expanded/contracted adaptation stimuli showed positive/negative $\triangle \mathrm{PSN}$ scores) across all eight one-sample t-tests (all $p<0.001$ ), suggesting that aftereffects were successfully induced in the predicted direction in all eight conditions.

In the ANOVA, as predicted a significant main effect of direction of adaptation was significant, $F(1,108)=177.72, p<.001, \eta p^{2}=.62$, with those exposed to expanded adaptation conditions showing positive $\triangle \mathrm{PSN}$ scores $(M=2.59, S D=1.83)$ and those in the contracted adaptation conditions showing negative $\triangle \mathrm{PSN}$ scores $(M=-1.33, S D=1.42)$. Unexpectedly however, there was a significant interaction between direction of adaptation and race of participant, $F(1,108)=14.60, p<.001, \eta p^{2}=.12$. Caucasian participants appeared to be experiencing stronger average body size aftereffects compared to the Asian participants in both expanded, $t(49.44)=-3.19$, $p=.002, d=.91($ Caucasian $M=3.27, S D=2.01 ;$ Asian $M=1.84, S D=1.26)$, and contracted, $t(57)$ $=2.16, p=.035, d=.57$ (Caucasian $M=-1.71, S D=1.33$; Asian $M=-.93, S D=1.42$ ) adaptation conditions. No other significant main effects or interactions were found (all ps > .05).

Importantly, there was no significant interaction found between race of test stimuli and race of adaptation stimuli, $F(1,108)=1.67, p=.199, \eta p^{2}=.02$, suggesting that transfer of aftereffects was complete across stimulus races. The lack of a significant result in a standard null-hypothesis significance test (NHST) should not, however, be taken as evidence of no difference between groups (absence of evidence is not evidence of absence). This result was therefore followed up with equivalence testing. This technique reverses the typical assumptions of NHST, setting the null 
hypothesis to 'there is a difference between the means', and the alternative hypothesis to 'the means are equivalent' (Lakens, 2017). The two one-sided tests (TOST) equivalence testing method was used to determine if the aftereffects were significantly equivalent in conditions where the race of the test stimuli was congruent vs conditions where the race of the test stimuli was incongruent with the race of the adaptation stimuli. Upper and lower bounds were set to $d_{z}=+/-.35$ (equivalent to a $+/$ $2.5 \%$ spherize transform - half of the difference between two adjacent frames in the transform sequence) (Lakens, 2017). The TOST procedure indicated that the observed effect size $\left(\mathrm{d}_{\mathrm{z}}=-.12\right)$ was significantly within the equivalent bounds, $\mathrm{t}(115)=2.65, \mathrm{p}=.030$.

\subsubsection{Discussion}

Experiment 1 found significant body size adaptation in the predicted directions in all conditions, such that exposure to expanded bodies resulted in an aftereffect of body size underestimation (i.e. more expanded bodies being perceived as normal), while exposure to contracted bodies resulted in body size overestimation (i.e. more contracted bodies being perceived as normal). The magnitude of the aftereffect was significantly equivalent in congruent and incongruent race conditions, suggesting that the transfer of adaptation across races of bodies was complete. Finally, Asian participants appeared to experience a significantly weaker body size adaptation effect compared to Caucasian participants, regardless of the race of the test and adaptation stimuli.

\subsection{Experiment 2}

Experiment 2 used a contingent adaptation paradigm, in which participants completed a method of adjustment task to determine the body size that they perceived as most "normal" (point of subjective normality; PSN) before and after exposure to adaptation bodies, which were either expanded Asians and contracted Caucasians or contracted Asians and expanded Caucasians. Following the results of Experiment 1, we predicted that there would be complete transfer of body 
size aftereffects across races of bodies, with a cancelling effect leading to much reduced or even absent aftereffects in both races of stimuli.

\subsubsection{Methods}

\subsubsection{Participants}

Forty five female participants were recruited in Sydney, Australia and Kota Kinabalu, Malaysia. Twenty four Australian Caucasian participants $\left(\mathrm{M}_{\mathrm{age}}=19.5, S D=2.25\right)$ were recruited in Sydney, Australia and 21 East/Southeast Asian Malaysian participants $\left(\mathrm{M}_{\mathrm{age}}=24.4, S D=2.25\right)$ were recruited in Kota Kinabalu, Malaysia in the same way as Experiment 1, giving 95\% power to detect a small-medium effect size for the hypothesised interaction in the ANOVA. Two Caucasian participants were excluded from the final analysis because they responded identically to every test stimulus.

\subsubsection{Stimuli}

The stimuli were the same as for Experiment 1.

\subsubsection{Procedure}

Experiment 2 employed a 2 (test race; within-subjects) x 2 (adaptation condition; between subjects) x 2 (participant race; between subjects) mixed factorial design. Participants were pseudorandomly assigned to one of two conditions, one in which participants were exposed to 5 expanded Asian and 5 contracted Caucasian adaptation stimuli, matched for initial BMI (A+/C-) and the other in which participants were exposed to 5 contracted Asian and 5 expanded Caucasian adaptation stimuli, matched for initial BMI (A-/C+), six times each for a total of two minutes. Top up stimuli in the adapted test phase (3 Asian and their initial BMI-matched Caucasian counterparts) were drawn at random from these adaptation stimuli each trial. All other aspects of the procedure were the same as Experiment 1. 


\subsubsection{Data processing and analysis}

$\triangle \mathrm{PSN}$ was calculated as in Experiment 1. Separate one-sample t-tests, for participants of each race, in each adaptation condition, and for test bodies of each race, were conducted comparing $\triangle \mathrm{PSN}$ values against a reference value of 0 to determine whether PSNs changed from baseline to adapted test phase. A 2 (adaptation condition) x 2 (race of test body) x 2 (participant race) mixed ANOVA was used to examine the effects of race on the magnitude and direction of the aftereffect.

For conditions in which the adaptation and test stimuli were incongruent, scores were multiplied by -1 , such that a positive result indicated an adaptation effect in the direction predicted if race-contingent adaptation were to occur. TOST was used to test for equivalence of effects between all congruent and incongruent conditions.

\subsubsection{Results}

No significant change in PSN was found for participants of either race, in either adaptation condition, for test bodies of either race (all $p s>.05$ ).

In the ANOVA, no significant main effects or interactions were found (all ps $>.05$ ), indicating that significant contingent aftereffects were not detected. The TOST procedure indicated that the observed effect size $\left(d_{z}=-0.06\right)$ was significantly within the equivalent bounds of $d_{z}=+/-0.35(+/-$ $4 \%$ of spherize transform - less than the difference between two adjacent frames in the transform sequence), $\mathrm{t}(42)=1.90, \mathrm{p}=0.032$, suggesting that aftereffects were of equivalent size in congruent and incongruent conditions.

\subsubsection{Discussion}

Participants did not show any significant change in their perception of normal body size after adaptation to stimuli of different races whose size had been manipulated in opposite directions. This result has a number of possible interpretations. Firstly, it is possible that no meaningful visual adaptation took place. However, given that this experiment was modelled on a previous investigation 
that showed significant contingent adaptation under highly similar conditions (Brooks et al, 2016), and given the significant adaptation effects detected in Experiment 1, this seems unlikely. An alternative explanation is that the neural populations processing body size for Asian and Caucasian bodies are largely or completely overlapping. In this case, the transfer of body size adaptation across races is predicted to be large or complete, as revealed by the significant equivalence test, such that the adaptation to the expanded and contracted bodies cancel each other out, to result in no net effect. This interpretation is consistent with the finding of no reduction in visual body size aftereffects for race-incongruent conditions in Experiment 1.

\section{General Discussion}

Across both experiments, participants showed equivalence in their adaptation to bodies of different races. In Experiment 1, when exposed to only expanded or contracted versions of only one race during the adaptation phase, participants exhibited body size adaptation effects in the same direction, and of equivalent magnitude in test stimuli of both races. It was noted, however, that Asian participants exhibited body size adaptation effects of reduced magnitude compared to Caucasian participants. In Experiment 2, participants showed no overall body size aftereffects when exposed to expanded Asian and contracted Caucasian adaptation bodies (or vice versa) in the same session, and aftereffects were equivalent for congruent and incongruent pairings of race of adaptation stimuli and race of test stimuli implying that, rather than resulting in simultaneous adaptation in opposite directions in different races, this exposure led to a cancellation effect.

A number of studies have demonstrated contingent adaptation for different race faces (Gwinn \& Brooks, 2015; Jaquet et al., 2008) and partial transfer of visual adaptation across faces of those same races (Jaquet et al., 2008), with authors inferring that the processing of faces of different races takes place in partially-dissociated neural populations. Similarly, partial transfer of body size aftereffects has been demonstrated across bodies of different identities (own- versus other-identity 
bodies), suggesting partial dissociation of the neural populations encoding body width in one's own versus other individuals' bodies (Brooks et al., 2016). However, our results suggest that body size aftereffects show complete transfer across bodies of different races, suggesting that a single, racegeneral neural population encodes the width of bodies of different races.

Contingent adaptation is dependent upon the classification of target stimuli into different groups (Little et al., 2008). The lack of race-contingent body size adaptation effects in the current studies may therefore reflect a lack of automatic categorisation of bodies by race. This lack of racial categorisation based on bodies is also reflected in studies examining the other-race effect (the wellknown phenomenon in which it is more difficult to recognise individuals of a different race than individuals of one's own race). While the other-race effect is well-established in faces (Hayden et al., 2007), similar effects can be induced in own-race faces by the induction of outgroup classification of faces, suggesting that outgroup classification may be necessary to induce the other-race effect (Zheng and Segalowitz, 2014). The other-race effect appears to be absent in bodies, however, providing further support for the suggestion that racial classification may not be automatic in bodies (Humphreys et al., 2005).

Unexpectedly, we found that Malaysian participants exhibited reduced body size adaptation effects compared to Australian participants across test and adaptation stimuli of both races. Further research is required to explain this phenomenon. However, we note that this novel finding fits with a broader pattern of differences between Caucasian and Asian participants in their performance on visual cognition tasks, including differences in the processing of faces (Blais et al., 2008), which some authors have attributed to broader cultural differences in cognitive styles (Blais et al., 2008). Our results suggest that racial/cultural differences in visual processing result in reduced susceptibility to visual adaptation among Asian populations. Although it is impossible to distinguish the environmental from the biological origins of these perceptual differences, it may be productive to 
investigate cultural differences in cognitive styles as an explanation for the origins of this difference in susceptibility to body size adaptation effects. One possible mechanism by which this may operate is that Asian participants may have directed more of their visual attention to the faces, and less to the bodies, than Caucasian participants, perhaps due to stronger cultural taboos against looking at bodies.

The body size aftereffect has been demonstrated to manipulate body misperception and dissatisfaction in quite negative ways across Western populations. Due to the pervasion of thin media images in modern Western, and increasingly Asian, society, understanding why individuals in certain cultures are less susceptible to this effect may have implications for interventions designed to reduce the harm associated with BSSM.

In conclusion, our results do not support the suggestion that the reduced body dissatisfaction seen in some non-Caucasian groups (DeBraganza and Hausenblas, 2010) can be explained by partial transfer of body size aftereffects from thin-idealised Caucasian bodies in the media to their own bodies. However, the unexpected demonstration of smaller body size aftereffects for Asian participants in general offers promise in providing a potential explanation for the reduced levels of body dissatisfaction shown in these cultures. 


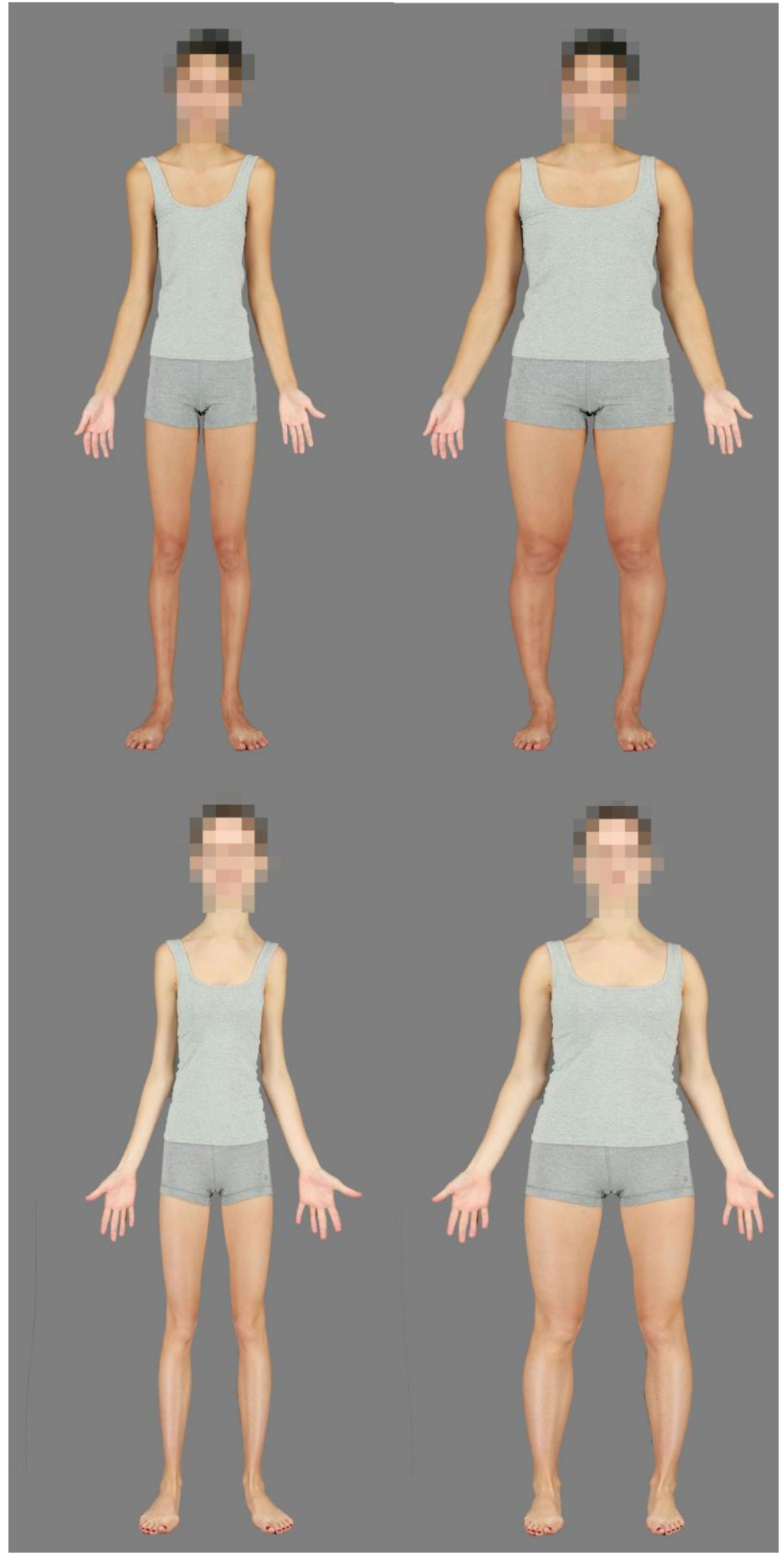

Figure 1: Examples of Asian (top) and Caucasian (bottom), contracted (left) and expanded (right) adaptation stimuli, matched on initial BMI. All faces were visible during experimentation 


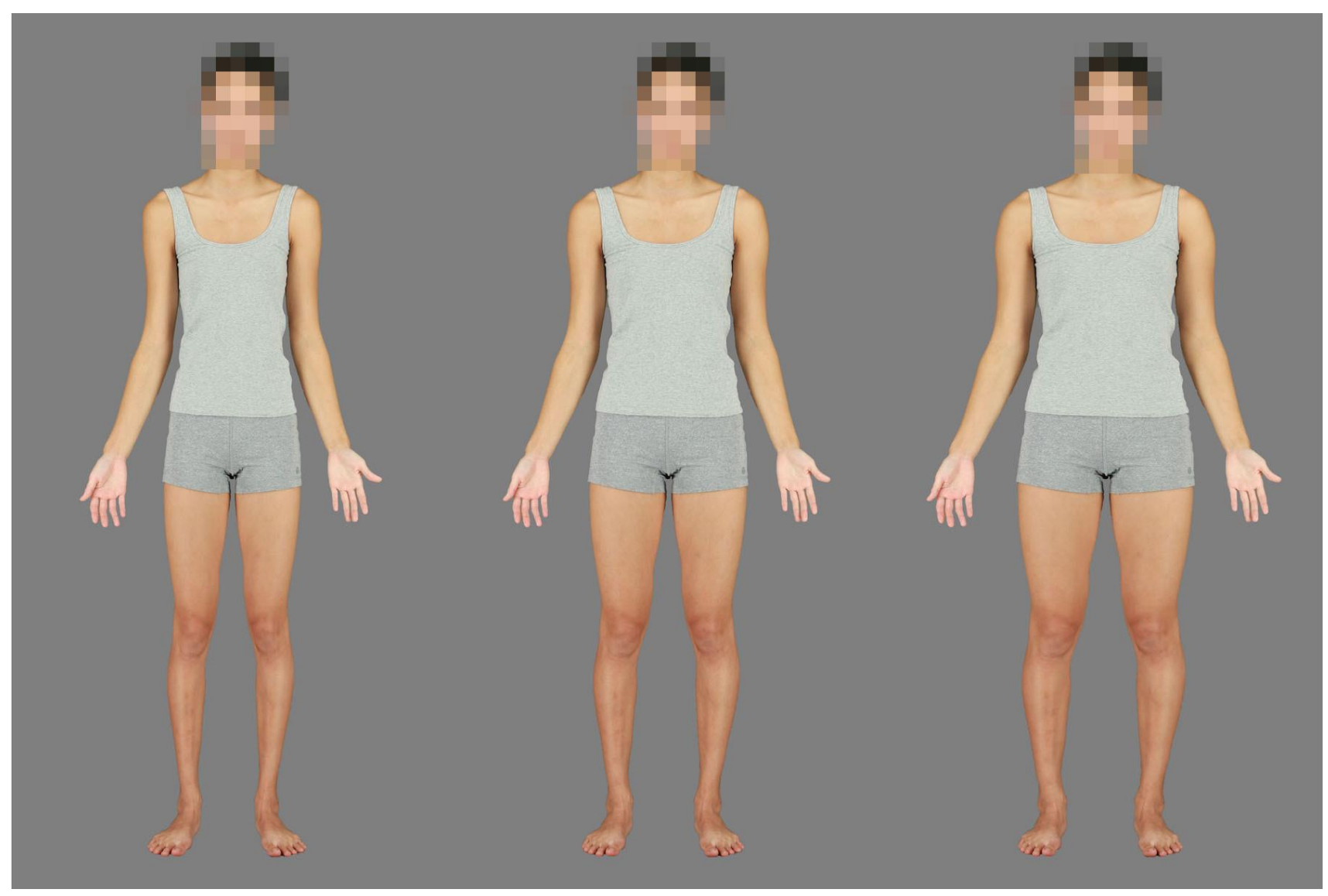

Figure 2: Original (centre) and contracted (left) and expanded (right) endpoints of the manipulation used to produce the test stimuli. All faces were visible during experimentation. 

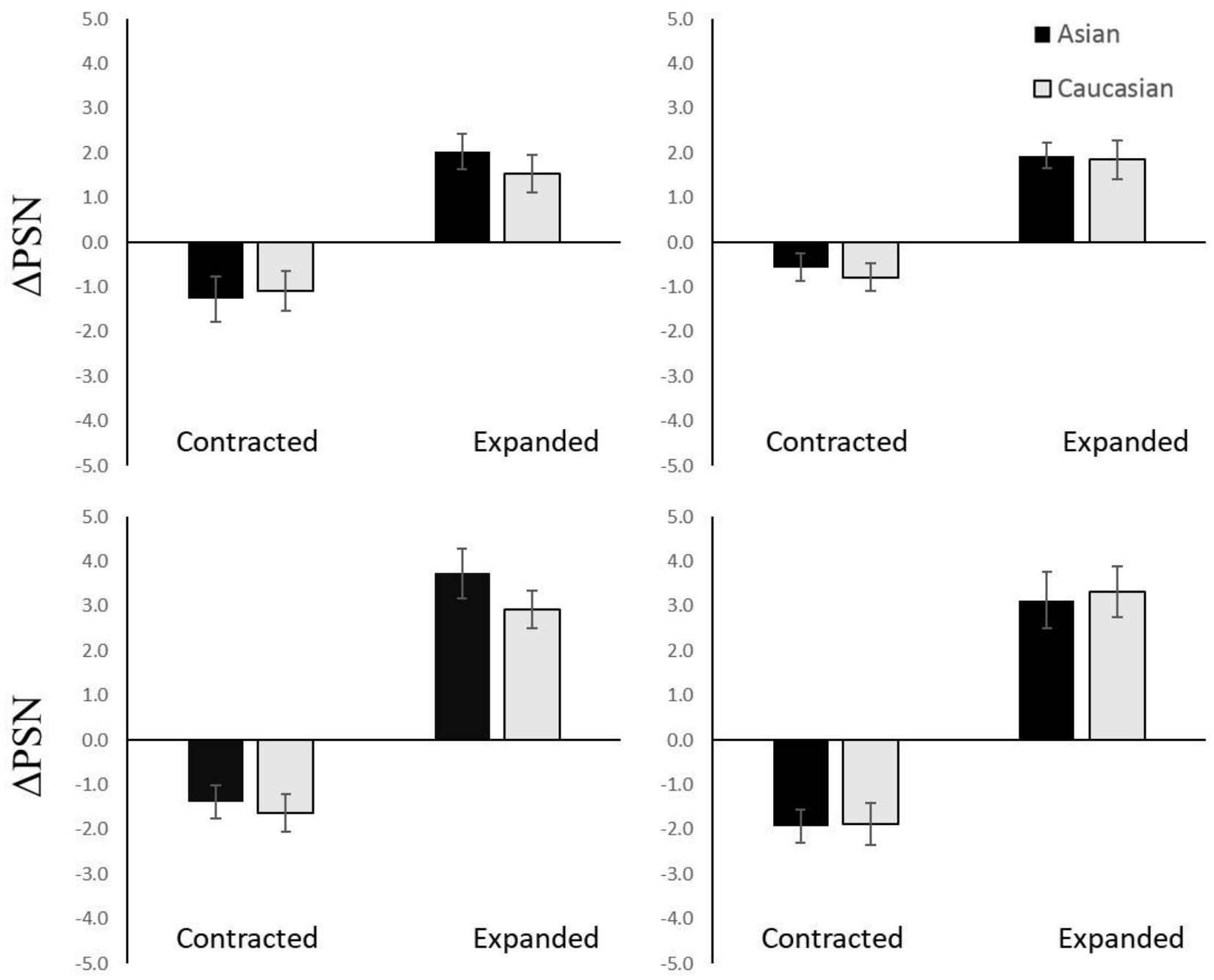

Figure 3. $\triangle P S N$ scores for Asian (top) and Caucasian (bottom) participants adjusting Asian (black bars) and Caucasian (light bars) test identities following exposure to Asian (left) and Caucasian (right) adaptation stimuli. Error bars show standard error of the mean.

\section{Conflicts of Interest}

The authors declare that the research was conducted in the absence of any commercial or financial relationships that could be construed as a potential conflict of interest.

\section{Author contributions}

LGF: Conception, design, collection, analysis and interpretation of data, drafting the article

CBYT: Collection of data, revision of article 
KRB: Conception, design, interpretation of data, revision of article

JM: Design, interpretation of data, revision of article

RJS: Interpretation of data, revision of article

IDS: Conception, design, collection, analysis and interpretation of data, drafting the article

\section{Funding}

No funding was received for this study.

\section{Datasets are available on request}

The raw data supporting the conclusions of this manuscript will be made available by the authors, to any qualified researcher.

\section{$4 \quad$ References}

Blais, C., Jack, R. E., Scheepers, C., Fiset, D., and Caldara, R. (2008). Culture shapes how we look at faces. PLoS One 3, e3022. doi:10.1371/journal.pone.0003022.

Boothroyd, L. G., Jucker, J. L., Thornborrow, T., Jamieson, M. A., Burt, D. M., Barton, R. A., et al. (2016). Television exposure predicts body size ideals in rural Nicaragua. Br. J. Psychol., 752767. doi:10.1111/bjop.12184.

Boothroyd, L. G., Tovée, M. J., and Pollet, T. V. (2012). Visual diet versus associative learning as mechanisms of change in body size preferences. PLoS One 7. doi:10.1371/journal.pone.0048691.

Bould, H., Carnegie, R., Allward, H., Bacon, E., Sapseid, M., Button, K., et al. (2018). Effects of visual adaptation on perception of and satisfaction with own body size: two randomised studies. R. Soc. Open Sci. doi:10.1016/S0924-977X(16)70056-3. 
Brooks, K. R., Mond, J. M., Stevenson, R. J., and Stephen, I. D. (2016). Body image distortion and exposure to extreme body types: Contingent adaptation and cross adaptation for self and other. Front. Neurosci. 10, 1-10. doi:10.3389/fnins.2016.00334.

Challinor, K. L., Mond, J., Stephen, I. D., Mitchison, D., Stevenson, R. J., Hay, P., et al. (2017). Body size \& shape misperception and visual adaptation: An overview of an emerging research paradigm. J. Int. Med. Res. 45, 2001-2008. doi:10.1177/0300060517726440.

DeBraganza, N., and Hausenblas, H. A. (2010). Media Exposure of the on Ideal Physique Women's Body Dissatisfaction and Mood. J. Black Stud. 40, 700-716.

Griffiths, S., Hay, P., Mitchison, D., Mond, J. M., McLean, S. A., Rodgers, B., et al. (2016). Sex differences in the relationships between body dissatisfaction, quality of life and psychological distress. Aust. N. Z. J. Public Health 40. doi:10.1111/1753-6405.12538.

Groesz, L. M., Levine, M. P., and Murnen, S. K. (2002). The effect of experimental presentation of thin media images on body satisfaction: A meta-analytic review. Int. J. Eat. Disord. 31, 1-16. doi:10.1002/eat.10005.

Gwinn, O. S., and Brooks, K. R. (2013). Race-contingent face aftereffects : A result of perceived racial typicality, not categorization. J. Vis. 13, 1-11. doi:10.1167/13.11.13.

Gwinn, O. S., and Brooks, K. R. (2015). Face encoding is not categorical: Consistent evidence across multiple types of contingent aftereffects. Vis. cogn. 6285, 1-27. doi:10.1080/13506285.2015.1091800.

Hayden, A., Bhatt, R. S., Joseph, J. E., and Tanaka, J. W. (2007). The Other-Race Effect in Infancy: Evidence Using a Morphing Technique. Infancy 12, 95-104. doi:10.1080/15250000701298923. 
Humphreys, G. W., Hodsoll, J., and Campbell, C. (2005). Attending but not seeing: The "other race" effect in face and person perception studied through change blindness. Vis. cogn. 12, 249-262. doi:10.1080/13506280444000148.

Jaquet, E., Rhodes, G., and Hayward, W. G. (2008). Race-contingent aftereffects suggest distinct perceptual norms for different race faces. Vis. cogn. 16, 734-753. doi:10.1080/13506280701350647.

Jucker, J. L., Thornborrow, T., Beierholm, U., Burt, D. M., Barton, R. A., Evans, E. H., et al. (2017). Nutritional status and the influence of TV consumption on female body size ideals in populations recently exposed to the media. Sci. Rep. 7, 1-9. doi:10.1038/s41598-017-08653-z.

Khor, G. L., Zalilah, M. S., Phan, Y. Y., Ang, M., Maznah, B., and Norimah, A. K. (2009). Perceptions of Body Image among Malaysian Male and Female Adolescents Perceptions of body image among Malaysian male and female adolescents. Singapore Med. J. 50, 303-311.

Lakens, D. (2017). Equivalence Tests : A Practical Primer for t Tests , Correlations , and MetaAnalyses. Soc. Psychol. Personal. Sci. 8, 355-362. doi:10.1177/1948550617697177.

Little, A. C., DeBruine, L. M., Jones, B. C., and Waitt, C. (2008). Category contingent aftereffects for faces of different races, ages and species. Cognition 106, 1537-1547. doi:10.1016/j.cognition.2007.06.008.

Mohr, H. M., Rickmeyer, C., Hummel, D., Ernst, M., and Grabhorn, R. (2016). Altered Visual Adaptation to Body Shape in Eating Disorders: Implications for Body Image Distortion. Perception. doi:10.1177/0301006616633385.

Powell, T. M., de Lemos, J. A., Banks, K., Ayers, C. R., Rohatgi, A. A., Khera, A., et al. (2010). Body size misperception: A novel determinent in the obesity epidemic. Arch. Intern. Med. 170, 
1695-1697. doi:10.1001/archinternmed.2010.314.

Quick, V., Nansel, T. R., Liu, D., Lipsky, L. M., Due, P., and Iannotti, R. J. (2015). Body size perception and weight control in youth: 9-year international trends from 24 countries. Int. J. Obes. 38, 988-994. doi:10.1038/ijo.2014.62.Body.

Rhodes, G., Jeffery, L., Evangelista, E., Ewing, L., Peters, M., and Taylor, L. (2011). Enhanced attention amplifies face adaptation. Vision Res. 51, 1811-1819. doi:10.1016/j.visres.2011.06.008.

Robinson, E., and Kirkham, T. C. (2014). Is he a healthy weight? Exposure to obesity changes perception of the weight status of others. Int. J. Obes. 38, 663-667. doi:10.1038/ijo.2013.154.

Schooler, D., Ward, L. M., Merriwether, A., and Caruthers, A. (2004). Who's that girl: Television's role in the body image development of young white and black women. Psychology 28, 38-47. doi:10.1111/j.1471-6402.2004.00121.x.

Stephen, I. D., and Perera, A. T.-M. (2014). Judging the difference between attractiveness and health: Does exposure to model images influence the judgments made by men and women? PLoS One 9, e86302. doi:10.1371/journal.pone.0086302.

Sturman, D., Stephen, I. D., Mond, J., Stevenson, R. J. R. J., and Brooks, K. R. (2017). Independent aftereffects of fat and muscle: Implications for neural encoding, body space representation, and body image disturbance. Nat. Sci. Reports 7, 40392. doi:10.1038/srep40392.

Thompson, P., and Burr, D. (2009). Visual aftereffects. Curr. Biol. 19, 11-14. doi:10.1016/j.cub.2008.10.014.

Wardle, J., Haase, A. M., and Steptoe, A. (2006). Body image and weight control in young adults: 
International comparisons in university students from 22 countries. Int. J. Obes. 30, 644-651. doi:10.1038/sj.ijo.0803050.

Yang, H., Shen, J., Chen, J., and Fang, F. (2011). Face adaptation improves gender discrimination. Vision Res. 51, 105-110. doi:10.1016/j.visres.2010.10.006.

Zheng, X., and Segalowitz, S. J. (2014). Putting a face in its place: In- and out-group membership alters the N170 response. Soc. Cogn. Affect. Neurosci. 9, 961-968. doi:10.1093/scan/nst069. 